\title{
Region-specific slowing of alpha oscillations is associated with visual-perceptual abilities in children born very preterm
}

\author{
Sam M. Doesburg 1,2,3, * Alexander Moiseev ${ }^{5}$, Anthony T. Herdman ${ }^{5,6}$, Urs Ribary $^{5,7,8,9}$ and Ruth E. Grunau ${ }^{8,9}$ \\ 1 Department of Diagnostic Imaging, The Hospital for Sick Children, Toronto, ON, Canada \\ ${ }^{2}$ Neurosciences \& Mental Health Program, Research Institute, The Hospital for Sick Children, Toronto, ON, Canada \\ ${ }^{3}$ Department of Medical Imaging, University of Toronto, Toronto, ON, Canada \\ ${ }^{4}$ Department of Psychology, University of Toronto, Toronto, ON, Canada \\ ${ }^{5}$ Behavioral and Cognitive Neuroscience Institute, Simon Fraser University, Burnaby, BC, Canada \\ ${ }^{6}$ Department of Audiology and Speech Sciences, The University of British Columbia, Vancouver, BC, Canada \\ 7 Department of Psychology, Simon Fraser University, Burnaby, BC, Canada \\ ${ }^{8}$ Developmental Neurosciences and Child Health, Child and Family Research Institute, Vancouver, BC, Canada \\ ${ }^{9}$ Department of Pediatrics, The University of British Columbia, Vancouver, BC, Canada
}

\section{Edited by:}

Christos Papadelis, Boston Children's Hospital, USA; Harvard Medical

School, USA

\section{Reviewed by:}

Paul Sauseng, University of Surrey, UK

Stephanie Jones, Brown University, USA

\section{*Correspondence:}

Sam M. Doesburg, Department of Diagnostic Imaging, The Hospital for Sick Children, 555 University Avenue, Toronto, ON M5G 1X8, Canada e-mail: sam.doesburg@sickkids.ca
Children born very preterm ( $\leq 32$ weeks gestational age) without major intellectual or neurological impairments often express selective deficits in visual-perceptual abilities. The alterations in neurophysiological development underlying these problems, however, remain poorly understood. Recent research has indicated that spontaneous alpha oscillations are slowed in children born very preterm, and that atypical alpha-mediated functional network connectivity may underlie selective developmental difficulties in visual-perceptual ability in this group. The present study provides the first source-resolved analysis of slowing of spontaneous alpha oscillations in very preterm children, indicating alterations in a distributed set of brain regions concentrated in areas of posterior parietal and inferior temporal regions associated with visual perception, as well as prefrontal cortical regions and thalamus. We also uniquely demonstrate that slowing of alpha oscillations is associated with selective difficulties in visual-perceptual ability in very preterm children. These results indicate that region-specific slowing of alpha oscillations contribute to selective developmental difficulties prevalent in this population.

Keywords: preterm, magnetoencephalography, neural oscillation, development, resting state, alpha-band, perception, cognition

\section{INTRODUCTION}

Children born very prematurely, even in the absence of brain injury and when intelligence is broadly normal, often experience selective developmental difficulties including problems with visual-perceptual abilities (Rickards et al., 2001; Grunau et al., 2002; Atkinson and Braddick, 2007). The biological basis of these issues remains poorly understood. MR imaging has identified numerous structural and functional atypicalities in very preterm children, many of which have been associated with problems in cognitive and perceptual development (Hart et al., 2008; Ment et al., 2009; Miller and Ferriero, 2009). An approach to understanding preterm child brain development which has received somewhat less attention is the mapping of neural oscillations using magnetoencephalography (MEG). MEG is a neurophysiological imaging modality that is particularly effective for characterizing the development of functional brain systems due to its unique combination of spatial and temporal resolution (Hari and Salmelin, 1997), and has been successfully employed to image brain activation in specific cortical systems in preterm infants and children (Nevalainen et al., 2008; Frye et al., 2010). Neural oscillations are known to be critical for brain activity and network connectivity supporting cognition and perception (Joliot et al.,
1994; Varela et al., 2001; Ward, 2003; Ribary, 2005; Uhlhaas et al., 2009a), and are altered in many clinical populations (Llinás et al., 1999; Schnitzler and Gross, 2005; Uhlhaas et al., 2009a), including those affecting child development (Murias et al., 2007; Mazaheri et al., 2010). Both spontaneous neural oscillations and their test-dependent dynamics develop throughout childhood and infancy (Clarke et al., 2001; Uhlhaas et al., 2009b; Xiang et al., 2009), including during the developmental epoch corresponding to very premature birth (Okumura et al., 2006; Gonzalez et al., 2011), and are relevant for the maturation of functional brain networks (Uhlhaas et al., 2010). Spontaneous cortical oscillations are characterized by a distinct peak in the alpha-band, and progressive increases in the frequency of spontaneous brain oscillations have been identified as a reliable marker of childhood neurodevelopment (John et al., 1980). Such developmental acceleration in alpha-band oscillations can be reliably measured using MEG. For example, maturational increases in the peak frequency of the mu rhythm have been reported in infants and school-age children (Berchicci et al., 2011) and deviations from typical patterns of spontaneous oscillations often indicate learning disabilities or increased risk for neurological disorders (Ahn et al., 1980). 
Using MEG, we previously demonstrated that spontaneous alpha oscillations $(8-14 \mathrm{~Hz})$ in school-age children born very preterm are slowed (Doesburg et al., 2011a). Atypical spontaneous alpha oscillations were also found to be associated with poor visual-perceptual abilities, and linked to extensive neonatal procedural pain, in children born at extremely low gestational age (Doesburg et al., 2013a). Such persistent alterations in the spectral structure of spontaneous brain oscillations may contribute to life-long cognitive difficulties in this group, as adults born at extremely low birth weight express altered ratio of high-frequency to low-frequency oscillations (Miskovic et al., 2009). Pronounced differences in long-range alpha-band MEG connectivity have also been identified in very preterm children during the performance of a visual short-term memory task, and these alterations were associated with selective visuospatial difficulties in this group (Doesburg et al., 2011b). Together, these findings indicate that spontaneous oscillations are slowed in very preterm children, and cortical alphaband connectivity dynamics supporting task processing are not typically expressed in this group. Our previous research has indicated that altered alpha oscillations are strongly related to selective difficulties with visual-perceptual abilities in very preterm children, rather than to general intellectual ability (Doesburg et al., 2011b, 2013a).

Previous studies of altered MEG alpha oscillations in very preterm children and their relation to cognitive development have analyzed data at the sensor level (Doesburg et al., 2011a,b, 2013a), which does not reveal the contribution of specific brain regions, thus limiting interpretation of the underlying functional systems involved. In the present study we investigated slowing of alpha oscillations in very preterm children within specific brain regions and examined their relation to difficulties in visual-perceptual abilities. To this end, we recorded spontaneous-eyes-open MEG activity from a group of school-age children born very preterm and full-term control children. Beamformer source analysis was employed to reconstruct brain activity from 72 locations distributed throughout the brain. These locations were predicated on an anatomical brain parcellation scheme in order to estimate activity within multiple functionally distinct brain regions. Peak oscillatory frequency was obtained for each analyzed brain region and compared with neuropsychological assessments.

\section{MATERIALS AND METHODS SUBJECTS}

Groups of 27 very preterm ( $\leq 32$ weeks GA) children and 27 fullterm controls were tested as part of a longitudinal study investigating the long-term impact of neonatal procedural pain on the neurocognitive development of very preterm children (i.e., Grunau et al., 2007, 2009). Full-term control children were recruited either through pediatricians in infancy or from the community at schoolage. The groups were matched on age, sex, and handedness. Both groups comprised 17 girls and 8 boys. Children had been excluded if they were diagnosed with a major sensory (hearing, vision), motor, or cognitive impairment, or had periventricular leukomalacia (PVL) or grade III-IV intraventricular hemorrhage (IVH) on neonatal ultrasound according to Papile's classification (Papile et al., 1978). Following inspection of movement during the MEG scan, one very preterm child, and one full-term control child were excluded due to excessive motion. The resulting group of 26 preterm children (mean age 7.76 years; $\mathrm{SD}=0.46$ years) consisted of 8 boys and 16 girls; the group of 26 full-term controls (mean age 7.66 years; SD $=0.28$ years) was comprised of 9 boys and 17 girls. Both groups contained 24 right handed children and 2 left handed children. The neonatal characteristics of the preterm group are presented in Table 1.

\section{MEG RECORDING}

Two minutes of spontaneous-eyes-open MEG activity was recorded using a 151-channel whole-head CTF Omega system (CTF Systems, Coquitlam, BC, Canada). Subjects were supine during recording and were instructed to maintain visual fixation on a "happy face" stimulus which was presented $40 \mathrm{~cm}$ above their eyes. A research assistant accompanied each subject within the magnetically shielded room to monitor the subjects. Data were digitized continuously at $1200 \mathrm{~Hz}$, stored offline for analysis, and subsequently downsampled to $600 \mathrm{~Hz}$. Fiducial coils were attached at the nasion as well as the right and left preauricular points, and each was energized at a distinct high narrow-band frequency. T1 weighted volumetric MRI images were also collected (1.5 T). Due to practical limitations imposed by multimodal neuroimaging in special child populations, MRI images were not available for all subjects. For those subjects without usable MRIs, a substitute matching MRI was found using the NIH database. MRIs for 4 very preterm children and 22 typically developing controls were taken from the NIH pediatric database. To obtain matching MRIs for individual subjects several candidate volumetric MRIs were selected from the database based on small differences between MEG and candidate MRI fiducial points. A best-matching structural MRI was then fitted to the subject's digitized head surface manually.

\section{MEG ANALYSIS}

Head motion was corrected for by obtaining a dipole source solution for each fiducial coil 30 times/s throughout the recording of spontaneous activity. The MEG data were then transformed to a common position by performing an inverse solution, data rotation, and forward solution 30 times/s (Wilson et al., 2007). To investigate slowing and reduction in the magnitude of spontaneous alpha oscillations in preterm children, we reconstructed the activity of multiple brain regions. The regions of interest for the source reconstruction were based on an anatomical brain parcellation scheme (see Kötter and Wanke, 2005; Bezgin et al., 2008).

\section{Table 1 | Neonatal characteristics of the very preterm group.}

\begin{tabular}{ll}
\hline Gestational age (weeks) & $29.82(2.17)$ \\
Birth weight $(\mathrm{g})^{*}$ & 1358.62 (402.68) \\
Singleton (\# subjects) & 18 \\
Early illness severity (SNAP-II)* & $10(11)$ \\
Days on mechanical ventilation* & $13(7)$ \\
IVH (Grade I-II; \# subjects) & 2
\end{tabular}

* One very preterm child was recruited from outside the longitudinal cohort, excluding scores for certain neonatal variables. 
This approach was chosen because it provides multiple functionally distinct regions which are distributed throughout the brain, and because this approach was successfully employed by a MEG study of functional brain activity and its relation to cognition, from which the set of source locations used in the present study were obtained (Diaconescu et al., 2011). A list of each source location is provided in Table 2, and a depiction of each seed location in brain space is available in Doesburg et al. (2013b). Each subject's MRI was warped into a common Talairach space using SPM2. The 72 locations were then warped back into each individual's brain space.

To reconstruct the activity from each analyzed source location, a minimum variance beamformer method was employed (Sekihara et al., 2001). This method has been shown to be very effective in estimating activity of a given source while maximally attenuating signal contributions from all other sources, as well as removing ocular and non-ocular artifacts (Cheyne et al., 2006, 2007). Note that on the sensor level volume currents result in mixing of brain signals from various locations. Source space analysis mostly eliminates the effects of volume currents, because the latter are already taken into account in the beamformer solution. Theoretically, minimum variance beamformers assume that correlations between sources are small, which might pose a problem when synchronous activity of different brain regions is expected. Extensive study of this question has shown that in practice the adverse effects of source correlations on the reconstructed power are significant only if correlations are very strong (Sekihara et al., 2002). Moreover, the signal-to-noise ratio (SNR) also needs to be relatively high for the correlations to introduce significant issues, such as SNRs observed in averaged evoked responses in event-related experimental paradigms. More advanced methods which take correlations into account show that low SNRs $(\sim 0.1)$, even when strong correlations exist $(\sim 0.8)$, do not alter reconstructed power significantly (Moiseev et al., 2011; Moiseev and Herdman, 2013). In the case of oscillatory resting state activity, correlations are moderate and SNRs are relatively small. It is for these regions that beamformer techniques have been successfully applied to resting state MEG data in recent years, including in functional connectivity analyses (Gross et al., 2001; Brookes et al., 2011; Hillebrand et al., 2012).

Data reconstructed from each of the 72 source locations were then filtered at $1 \mathrm{~Hz}$ intervals from 1 to $60 \mathrm{~Hz}$ (passband $=f \pm 0.05 f$, where $\mathrm{f}$ is the filter frequency), using the eegfilt function from the EEGLAB toolbox, a two-way least-squares FIR filter (Delorme and Makeig, 2004). These methods which has previously been effective for estimating the spectral content of oscillatory signals in M/EEG data (Doesburg et al., 2008, 2011a,b). Power was calculated at each frequency for each data point and sensor, and was subsequently averaged across all time points in the $120 \mathrm{~s}$ recording session. Peak oscillatory frequency for each brain region for each subject was defined as the frequency expressing maximum power between 5 and $20 \mathrm{~Hz}$, which included the alphaband $(8-14 \mathrm{~Hz})$, as well as other nearby frequencies. This wider frequency range was selected because peak frequencies of MEG oscillations in specific brain regions in atypically developing children have not been extensively studied. Given this, we did not wish to make strong a priori assumptions about frequency content in specific brain regions in very preterm children. Furthermore, slowing of alpha rhythms into the upper theta range has been reported in several other clinical populations (Llinás et al., 1999; Sarnthein et al., 2006; Sarnthein and Jeanmonod, 2007, 2008; Boord et al., 2008). These methods for determining peak oscillatory frequency in MEG data have been previously established (Doesburg et al., 2011a).

To test the hypothesis that peak oscillatory frequency is slowed in very preterm children without statistical challenges imposed by multiple comparisons, peak frequency was averaged across all 72 analyzed regions for each subject to obtain a global measure for each subject. Permutation testing was then used to test for global differences between the very preterm and full-term control groups (see Blair and Karniski, 1993). Permutation tests were one-tailed as the direction these effects were predicted from previous results (Doesburg et al., 2011a). Once global group differences were established we tested whether oscillatory slowing was present in each of the 72 analyzed brain regions. Since our goal at this stage of the analysis was to determine which set of regions contributed to the established global pattern of oscillatory slowing, and type II errors are as misleading as type I errors in determining which brain regions are involved in the global pattern and which are not, these tests were not corrected for multiple comparisons. The rationale for this is that the conservative nature of such corrections would likely underestimate the number of areas expressing atypical alpha oscillations and provide a distorted account of the location and extent of altered brain oscillations in very preterm children. Permutation tests were also used to evaluate regional between-group comparisons.

\section{ASSOCIATIONS BETWEEN MEG ACTIVITY AND PSYCHOMETRIC DATA}

Following MEG recording, all children underwent psychometric assessment using the Wechsler Intelligence Scale for Children, 4th Edn. (WISC-IV; Wechsler, 2003) and the Beery-Buktenica Developmental Test of Visual-Motor Integration, 5th Edn. (Beery et al., 2004), administered by a psychometrician. For the present study, we elected to focus on visual-perceptual abilities as previous studies have indicated that altered alpha oscillations in preterm children are strongly associated with visual-perceptual difficulties (Doesburg et al., 2011b, 2013a). The visual perception subscale of the Beery VMI was chosen as an index of visual-perceptual ability, as it has proven to be a sensitive measure in previous studies (Doesburg et al., 2011b, 2013a). The Motor Coordination and Visual-Motor Integration Subscales of the Beery VMI and FullScale IQ (FSIQ) from the WISC-IV were also evaluated in relation to the MEG data in order to assess the specificity of relations between altered alpha oscillations and functional abilities in very preterm children, consistent with methods employed in previous research (Doesburg et al., 2011a).

Correlations were examined between each of these psychometric measures, within both the very preterm and full-term groups, and peak oscillatory frequency for each brain region that exhibited slowing. Statistical analysis of correlations between oscillatory slowing and psychometric data were controlled for multiple comparisons using the False Discovery Rate (FDR; see Storey, 2002). The approach taken to multiple comparisons here differs from that in the regional analysis of oscillatory slowing because correlations among oscillatory brain activity and psychometric measures aim 
Table 2 | Each source location used in the analysis of spontaneous MEG activity, together with associated Brodmann area and Talairach coordinates.

\begin{tabular}{|c|c|c|c|c|c|}
\hline Source & BA & Hemisphere & $x$ & $\boldsymbol{Y}$ & $Z$ \\
\hline Anterior cingulate cortex & 32 & Midline & 0 & 32 & 24 \\
\hline Posterior cingulate cortex & 23 & Midline & 0 & -32 & 24 \\
\hline Retrosplenial cingulate cortex & 30 & Midline & 0 & -48 & 12 \\
\hline Subgenual cingulate cortex & 25 & Midline & 0 & 16 & -8 \\
\hline A1 (primary auditory) & & Left & -40 & -14 & 4 \\
\hline A2 (secondary auditory) & 22 & Left & -60 & -14 & 4 \\
\hline Frontal eye fields & 6 & Left & -36 & 8 & 56 \\
\hline Anterior insula & 13 & Left & -36 & 16 & -4 \\
\hline Claustrum & & Left & -36 & -8 & -4 \\
\hline M1 (primary motor) & 4 & Left & -24 & -24 & 56 \\
\hline Inferior parietal cortex & 40 & Left & -44 & -48 & 20 \\
\hline Angular gyrus & 39 & Left & -44 & -64 & 28 \\
\hline Precuneus & 7 & Left & -8 & -64 & 54 \\
\hline Superior parietal cortex & 7 & Left & -28 & -56 & 54 \\
\hline Centrolateral prefrontal cortex & 46 & Left & -48 & 32 & 12 \\
\hline Dorsolateral prefrontal cortex & 9 & Left & -48 & 36 & 32 \\
\hline Dorsomedial prefrontal cortex & 8 & Left & -8 & 36 & 40 \\
\hline Medial prefrontal cortex & 10 & Left & -8 & 48 & 20 \\
\hline Orbitofrontal cortex & 11 & Left & -24 & 44 & -20 \\
\hline Frontal polar & 10 & Left & -24 & 64 & 4 \\
\hline Ventrolateral prefrontal cortex & & Left & -48 & 32 & -8 \\
\hline Parahippocampal cortex & & Left & -28 & -16 & -16 \\
\hline Dorsolateral premotor cortex & 6 & Left & -28 & 0 & 60 \\
\hline Medial premotor cortex & 6 & Left & -4 & 0 & 60 \\
\hline Ventrolateral premotor cortex & 9 & Left & -44 & 4 & 24 \\
\hline Pulvinar & & Left & -16 & -28 & 4 \\
\hline S1 (primary somatosensory) & 3 & Left & -40 & -28 & 64 \\
\hline S2 (secondary somatosensory) & 43 & Left & -56 & -16 & 16 \\
\hline Middle temporal cortex & 21 & Left & -64 & -24 & -12 \\
\hline Inferior temporal cortex & 20 & Left & -64 & -24 & -24 \\
\hline Temporal pole & 38 & Left & -52 & 12 & -28 \\
\hline Superior temporal cortex & 22 & Left & -52 & -4 & -8 \\
\hline Ventral temporal cortex & & Left & -32 & -28 & -28 \\
\hline Thalamus (ventral lateral nucleus) & & Left & -8 & -8 & 4 \\
\hline V1 (primary visual) & & Left & -4 & -84 & -4 \\
\hline V2 (secondary visual) & & Left & -4 & -96 & 8 \\
\hline Cuneus & 18 & Left & -20 & -88 & 20 \\
\hline Fusiform gyrus & 19 & Left & -20 & -84 & -12 \\
\hline A1 (primary auditory) & & Right & 40 & -14 & 4 \\
\hline A2 (secondary auditory) & 22 & Right & 60 & -14 & 4 \\
\hline Frontal eye fields & 6 & Right & 36 & 8 & 56 \\
\hline Anterior insula & 13 & Right & 36 & 16 & -4 \\
\hline Claustrum & & Right & 36 & -8 & -4 \\
\hline M1 (primary motor) & 4 & Right & 24 & -24 & 56 \\
\hline Inferior parietal cortex & 40 & Right & 44 & -48 & 20 \\
\hline Angular gyrus & 39 & Right & 44 & -64 & 28 \\
\hline Precuneus & 7 & Right & 8 & -64 & 54 \\
\hline Superior parietal cortex & 7 & Right & 28 & -56 & 54 \\
\hline Centrolateral prefrontal cortex & 46 & Right & 48 & 32 & 12 \\
\hline Dorsolateral prefrontal cortex & 9 & Right & 48 & 36 & 32 \\
\hline
\end{tabular}

(Continued)

\begin{tabular}{lrlccc}
\hline Source & BA & Hemisphere & $\boldsymbol{X}$ & $\boldsymbol{Y}$ & $\boldsymbol{Z}$ \\
\hline Dorsomedial prefrontal cortex & 8 & Right & 8 & 36 & 40 \\
Medial prefrontal cortex & 10 & Right & 8 & 48 & 20 \\
Orbitofrontal cortex & 11 & Right & 24 & 44 & -20 \\
Frontal polar & 10 & Right & 24 & 64 & 4 \\
Ventrolateral prefrontal cortex & & Right & 48 & 32 & -8 \\
Parahippocampal cortex & & Right & 28 & -16 & -16 \\
Dorsolateral premotor cortex & 6 & Right & 28 & 0 & 60 \\
Medial premotor cortex & 6 & Right & 4 & 0 & 60 \\
Ventrolateral premotor cortex & 9 & Right & 44 & 4 & 24 \\
Pulvinar & & Right & 16 & -28 & 4 \\
S1 (primary somatosensory) & 3 & Right & 40 & -28 & 64 \\
S2 (secondary somatosensory) & 43 & Right & 56 & -16 & 16 \\
Middle temporal cortex & 21 & Right & 64 & -24 & -12 \\
Inferior temporal cortex & 20 & Right & 64 & -24 & -24 \\
Temporal pole & 38 & Right & 52 & 12 & -28 \\
Superior temporal cortex & 22 & Right & 52 & -4 & -8 \\
Ventral temporal cortex & & Right & 32 & -28 & -28 \\
Thalamus (ventral lateral nucleus) & & Right & 8 & -8 & 4 \\
V1 (primary visual) & & Right & 4 & -84 & -4 \\
V2 (secondary visual) & & Right & 4 & -96 & 8 \\
Cuneus & 18 & Right & 20 & -88 & 20 \\
Fusiform gyrus & 19 & Right & 20 & -84 & -12 \\
\hline & & & & & \\
\hline
\end{tabular}

Table adapted from Diaconescu et al. (2011).

to determine whether such relationships exist, rather than characterize the location and extent of phenomena which have already been established as statistically significant on a global level.

\section{RESULTS}

\section{OSCILLATORY SLOWING IN CHILDREN BORN VERY PRETERM}

Analysis of global peak oscillatory frequency (averaged across all 72 analyzed brain regions) revealed statistically significant slowing in school-age children born very prematurely $(p=0.021)$. Regional analysis of oscillatory slowing revealed that this pattern was widespread and encompassed prefrontal, temporal, and parietal areas, as well as aspects of thalamus and cingulate cortex. Complete results of the analysis of regional oscillatory slowing in very preterm children are presented in Figure 1. To summarize, slowing was widespread in bilateral temporal cortex, including bilateral in temporal areas implicated in the ventral visual processing stream (i.e., ventral and inferior temporal cortex). Parietal regions associated with visual processing were also implicated (i.e., bilateral inferior parietal cortex). Slowing was also present in secondary somatosensory cortex in both hemispheres, and also encompassed numerous in prefrontal regions (i.e., centrolateral, dorsolateral, ventrolateral, and orbitofrontal cortex).

\section{OSCILLATORY SLOWING ASSOCIATED WITH REDUCED} VISUAL-PERCEPTUAL ABILITY IN VERY PRETERM CHILDREN

Visual-perceptual ability, indexed by the visual perception subscale of the Beery VMI, was associated with oscillatory slowing 


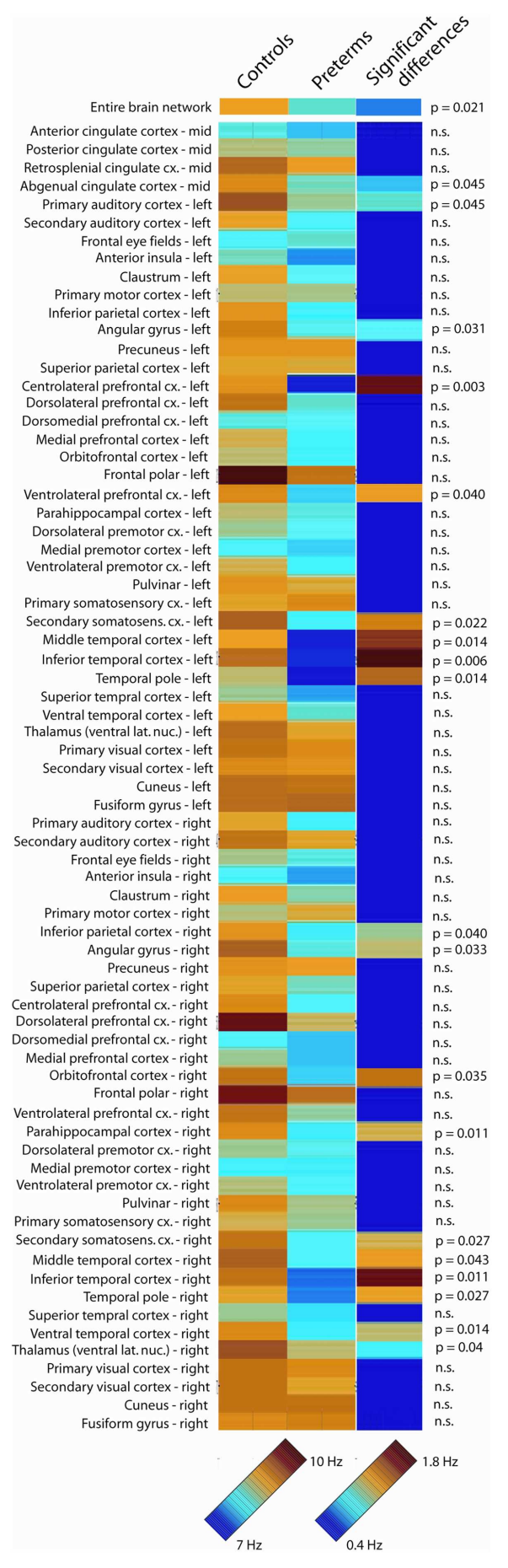

FIGURE 1 | Oscillatory slowing in very preterm children. Peak oscillatory frequency in each of the 72 analyzed brain regions for the full-term control and very preterm groups, as well as statistically significant regional slowing in children born very preterm. The $7-$ to $10-\mathrm{Hz}$ scale bar corresponds to the peak oscillatory frequency for each analyzed region for the very preterm and typically developing groups. The $0.4-$ to $1.8-\mathrm{Hz}$ scale bar indicates the difference in peak oscillatory frequency between the very preterm and typically developing groups for regions where statistically significant differences were observed (regions for which no significant group differences were observed are presented in dark blue). in multiple regions, including several known to be involved in the processing of visual information including inferior parietal cortex, ventral temporal cortex, and thalamus. Regional slowing was not significantly associated with FSIQ or the Motor or Visual-Motor scores on the Beery VMI for the very preterm children. Peak oscillatory frequency was not associated with any of the psychometric tests for full-term children for any region. Complete results of the analysis of associations between regional oscillatory slowing and visual-perceptual ability in preterm children are provided in Table 3.

\section{DISCUSSION}

We provide the first source-resolved analysis of slowing of alpha oscillations in children born very preterm. Identification of the specific brain regions across thalamocortical systems involved in slowed alpha oscillations in very preterm children represents an important step, as altered alpha oscillations may mediate relations between neonatal procedural pain and cognitive outcome in children born at extremely low gestational age (Doesburg et al., 2013a), and alpha oscillations are particularly relevant for altered network connectivity underlying problems in visual-perceptual abilities in very preterm children (Doesburg et al., 2011b). We observed oscillatory slowing within multiple cortical regions encompassing bilateral temporal, parietal, and prefrontal cortical areas. Many regions expressing oscillatory slowing in preterm children are important for visual processing, including regions involved in both the dorsal visual system (i.e., inferior parietal cortex), and the ventral visual system (i.e., inferior temporal cortex, ventral temporal

Table 3 | Associations between slowing of peak oscillatory frequency and visual-perceptual ability, indexed by the visual perception subscore of the Beery VMI, in children born very preterm.

\begin{tabular}{|c|c|c|}
\hline Source & $\begin{array}{l}\text { Correlation } \\
\text { coefficient }\end{array}$ & $\begin{array}{l}P \text { value } \\
\text { (FDR corrected) }\end{array}$ \\
\hline Subgenual cingulate cortex (midline) & 0.44 & 0.028 \\
\hline Primary auditory cortex (left) & n.s. & n.s. \\
\hline Angular gyrus (left) & 0.53 & 0.019 \\
\hline Centrolateral prefrontal cortex (left) & n.s. & n.s. \\
\hline Ventrolateral prefrontal cortex (left) & n.s. & n.s. \\
\hline Secondary somatosensory cortex (left) & n.s. & n.s. \\
\hline Middle temporal cortex (left) & n.s. & n.s. \\
\hline Inferior temporal cortex (left) & n.s. & n.s. \\
\hline Temporal pole (left) & n.s. & n.s. \\
\hline Inferior parietal cortex (right) & 0.44 & 0.037 \\
\hline Angular gyrus (right) & n.s. & n.s. \\
\hline Orbitofrontal cortex (right) & 0.44 & 0.031 \\
\hline Parahippocampal cortex (right) & 0.47 & 0.038 \\
\hline Secondary somatosensory cortex (right) & n.s. & n.s. \\
\hline Middle temporal cortex (right) & n.s. & n.s. \\
\hline Inferior temporal cortex (right) & n.s. & n.s. \\
\hline Temporal pole (right) & n.s. & n.s. \\
\hline Ventral temporal cortex (right) & 0.54 & 0.035 \\
\hline Thalamus (right ventral lateral nucleus) & 0.45 & 0.038 \\
\hline
\end{tabular}

Significant correlations are presented in bold. 
cortex). Slowing of alpha oscillations in preterm children was also prevalent in areas of prefrontal cortex involved in executive function (i.e., dorsolateral prefrontal cortex and orbitofrontal cortex).

The present study provides the first evidence that slowing of peak oscillatory frequency is associated with cognitive outcome in very preterm children. Oscillatory slowing was found to be selectively related to visual-perceptual ability, an area of selective developmental vulnerability in very preterm children (Rickards et al., 2001; Grunau et al., 2002; Taylor et al., 2004), and not associated with overall intellectual function, indexed by FSIQ. These results confirmed associations between slowing of alpha oscillations and visual-perceptual ability were typically, but not exclusively, found in regions involved in visual processing including inferior parietal cortex, angular gyrus, ventral temporal, cortex, and thalamus. There were no associations found between peak oscillatory frequency and neuropsychological ability in the fullterm children, indicating that this phenomenon corresponds to neural mechanisms underlying selective developmental difficulties prevalent in very preterm children, rather than normal variance in childhood neurocognitive development.

Although not as prevalent as was observed for visual cortical regions, oscillatory slowing was also present in prefrontal cortical areas (i.e., ventrolateral prefrontal cortex and orbitofrontal cortex), congruent with earlier reports of slowed alpha oscillations over frontal MEG sensors (Doesburg et al., 2011a). This may be significant as executive functions are also an area of selective developmental difficulty prevalent in children born very prematurely (Anderson et al., 2004; Marlow et al., 2007; Mulder et al., 2009). Together with our results implicating region-specific slowing of alpha oscillations with selective difficulties in visual-perceptual abilities, the presence of atypical alpha rhythmicity in prefrontal brain regions in very preterm children suggests that region-specific slowing may be associated with developmental difficulties in executive function as well. Stated more generally, alpha slowing within a specific cortical region may be associated with reduced function in the corresponding psychological domain. In this view, specific cortical systems situated in posterior parietal, inferior temporal, and prefrontal regions may be selectively vulnerable to aspects of adverse neonatal experience, impacting the development of brain oscillations underlying the maturation of cognitive and perceptual functions associated with these areas (Benasich et al., 2008; Uhlhaas et al., 2009b, 2010) and causing selective developmental difficulties in the corresponding domains of executive function and visual-perceptual ability.

The perspective that oscillatory slowing is associated with impairment of normal brain function is consistent with observations of MEG slowing in association with neurological insults in other contexts. For example, regional analysis of excessive lowfrequency oscillations has been introduced as a method for mapping abnormal functional brain activity following mild traumatic brain injury (Huang et al., 2012), and the anatomical focus of such excessive slow wave activity appears related to the location of white matter injury, measured using diffusion tensor imaging, in this population (Huang et al., 2009). Furthermore, slowing of peak alpha oscillatory frequency toward the theta range in resting state MEG recordings has been reported in diverse pathological conditions including Parkinson's disease, neurogenic pain, tinnitus, and major depression (Llinás et al., 1999). Subsequent combination of methods for the analysis of oscillatory slowing with MEG source analysis techniques have indicated that neuroanatomical systems involved in slowing depend on the pathological condition in question, and likely correspond to the nature of symptoms expressed (Schulman et al., 2011). This finding is consistent with the results of the present study which demonstrates that regionspecific slowing of alpha oscillations in visual cortical regions are associated with visual-perceptual difficulties in children born preterm. Slowing of alpha oscillations in neuropathic pain has been confirmed using scalp EEG (Sarnthein et al., 2006; Boord et al., 2008). Slowing of alpha oscillations toward the theta range has also been reported using intracranial EEG in patients with neuropathic pain and Parkinson's disease (Sarnthein and Jeanmonod, $2007,2008)$. Oscillatory alpha slowing in M/EEG recordings in numerous pathological conditions is thought to result from deafferentation, particularly in thalamocortical systems (Llinás et al., 2005), and it has been demonstrated that the parameters of alpha oscillations are related to white matter properties revealed using diffusion tensor imaging (Valdés-Hernández et al., 2010).

Slowing of alpha oscillations, together with the purported association of this phenomenon with the loss of integrity in structural brain connectivity, raises questions regarding relations between alpha oscillatory slowing and communication in brain networks. In particular, evidence increasingly indicates that coherence of alpha-band oscillations plays a critical role in communication among brain regions supporting cognition and perception (see Palva and Palva, 2007, 2012 for reviews). Recently, it has been reported that alpha-band oscillatory connectivity is reduced in preterm neonates with brain lesions (Tokariev et al., 2012). Alphaband oscillatory connectivity during cognitive processing has been found to be slowed and reduced, and associated with selective difficulties in visual-perceptual ability, in very preterm children (Doesburg et al., 2011b). Moreover, this reduced alpha-band phase synchronization was observed in concert with increased interhemispheric theta-band synchronization in the very preterm children, suggesting that task-dependent interactions among brain regions may also be slowed. In light of the prevalence of white matter injury and atypical white matter development in this population (see Khwaja and Volpe, 2008; Miller and Ferriero, 2009), this raises the prospect that increased conduction delays among cortical regions may adversely impact the ability to recruit inter-regional oscillatory network coherence to support task performance.

The observation that slowing of alpha-band oscillations in very preterm children occurred in thalamus is also significant as animal research has demonstrated that thalamocortical mechanisms underlie the generation of alpha oscillations, as well as their slowing under pathological conditions (see Hughes and Crunelli, 2005 for review). Moreover, thalamocortical systems undergo critical phases of development during the gestational epoch corresponding to very premature birth (see Kostovic and Judaš, 2010 for review). The slowing of alpha oscillations, mediated by altered thalamocortical interactions, has been proposed to underlie disruptions of function in several neurological and neuropsychiatric populations (Llinás et al., 1999, 2005). Altered connectivity and white matter development are prevalent in children born very preterm (see Miller and Ferriero, 2009 for review), including 
altered development of structural (Anjari et al., 2007; Dudink et al., 2007) and functional (Smyser et al., 2010) connectivity in thalamocortical systems. The importance of thalamus for understanding alterations in cortical development in preterm children is further underscored by recent findings that reduced thalamic volume is predictive of reduced cortical volume in preterm infants (Ball et al., 2012).

Our results showing significant slowing in deep brain structures such as the thalamus should be interpreted with caution. Reconstructing MEG signals from deep structures is not only difficult, but in many cases impossible because the SNR is too small (see Quraan et al., 2011). It should be noted, however, that in terms of power the alpha-band signal is the strongest in the brain, improving the likelihood of accurate source reconstruction in this frequency range (Attal et al., 2007). Moreover, prior MEG source analysis studies have reported task-dependent changes involving thalamus (i.e., Schnitzler et al., 2009). Further investigation will be needed to definitively determine the reliability of MEG measures of oscillations power from deep structures such as thalamus. Future research will also be required to examine relations between the structural and functional development of thalamocortical systems, disruption of the normative structure of cortical alpha oscillations, and functional outcomes in specific psychological domains in children who were born very prematurely. Involvement of specific regions identified in the source space analysis of oscillatory slowing in very preterm children should also be interpreted with some caution, as correction for multiple comparisons was not performed across all analyzed 72 regions. As such, potential false positives for individual regions may have arisen. The consistency of slowing located in regions involved in dorsal and ventral visual pathways, however, together with prefrontal cortical regions, is highly suggestive that region-specific slowing in brain structures is associated with selective developmental difficulties prevalent in children born very prematurely.

\section{CONCLUSION}

We demonstrate that slowing of spontaneous alpha oscillations is evident in widespread brain regions in very preterm children. These alterations of alpha oscillations were concentrated in regions involved in visual processing including thalamus, posterior parietal cortex, and interior temporal cortex, as well as in prefrontal regions relevant for executive functions. We provide the first evidence that slowing of spontaneous alpha oscillations is associated with functional outcome in preterm children. This association between slowing of alpha oscillations in particular brain regions and function was specific for visual-perceptual abilities, and was typically found in posterior parietal and inferior temporal brain regions implicated in visual processing. These results indicate that area-specific atypicalities in alpha oscillatory brain activity are associated with selective difficulties in children born very prematurely.

\section{ACKNOWLEDGMENTS}

We would like to thank Dr. Ivan Cepeda and Gisela Gosse for coordinating the study, Katia Jitlina, Amanda Degenhardt, Cecil Chau, Teresa Cheung, and Julie Unterman for their help in data collection. This work was supported by grant RO1 HD039783 from The Eunice Kennedy Shriver National Institute of Child Health and Human Development of the National Institutes of Health (NICHD/NIH) to Ruth E. Grunau, who received salary support from the Child and Family Research Institute (CFRI). Urs Ribary holds a BC LEEF Leadership Chair in Cognitive Neuroscience in Early Childhood Health and Development supported by the BC Leading Edge Endowment Fund (BC LEEF), and the MEG facility infrastructure was supported by the CFI grant to Urs Ribary and the Behavioral and Cognitive Neuroscience Institute (BCNI). Research support for Sam M. Doesburg is from the Natural Sciences and Engineering Research Council of Canada (NSERC RGPIN 435659) and the EpLink program of the Ontario Brain Institute (OBI).

\section{REFERENCES}

Ahn, H., Prichep, L., John, E. R., Baird, H., Trepetin, M., and Kaye, H. (1980). Developmental equations reflect brain dysfunctions. Science 210, 1259-1262. doi:10.1126/science.7434027

Anderson, P. J., Doyle, L. W., and Victorian Infant Collaborative Study Group. (2004). Executive functioning in school-aged children who were born very preterm or with extremely low birth weight in the 1990s. Pediatrics 114, 50-57. doi:10.1542/peds.114.1.50

Anjari, M., Srinivasan, L., Allsop, J. M., Hajnal, J. V., Rutherford, M. A., Edwards, A. D., et al. (2007). Diffusion tensor imaging with tract-based spatial statistics reveals local white matter abnormalities in preterm infants. Neuroimage 35, 1021-1027. doi:10.1016/j.neuroimage.2007.01.035

Atkinson, J., and Braddick, O. (2007). Visual and visuocognitive development in children born very prematurely. Prog. Brain Res. 164, 123-149. doi:10.1016/S00796123(07)64007-2

Attal, Y., Bhattacharjee, M., Yelnik, J., Cottereau, B., Lefevre, J., Okada, Y., et al. (2007). Modeling and detecting deep brain activity with MEG and EEG. Conf. Proc. IEEE Eng. Med. Biol. Soc. 2007, 4937-4940. doi:10.1109/IEMBS.2007.4353448

Ball, G., Boardman, J. P., Rueckert, D., Aljabar, P., Arichi, T., Merchant, N., et al. (2012). The effect of preterm birth on thalamic and cortical development. Cereb. Cortex 22, 1016-1024. doi:10.1093/cercor/bhr176

Beery, K. E., Buktenica, N. A., and Beery, N. A. (2004). Beery-Buktenica Developmental Test of Visual-Motor Integration, 5th Edn. San Antonio: Psychological Corporation.

Benasich, A. A., Gou, Z., Choudhury, N., and Harris, K. D. (2008). Early cognitive and language skills are linked to resting frontal gamma power across the first 3 years. Behav. Brain Res. 195, 215-222. doi:10.1016/j.bbr.2008.08.049

Berchicci, M., Zhang, T., Romero, L., Peters, A., Annett, R., Teuscher, U., et al. (2011). Development of mu rhythm in infants and preschool children. Dev. Neurosci. 33, 130-143. doi:10.1159/000329095

Bezgin, G., Wanke, E., Krumnack, A., and Kotter, R. (2008). Deducing logical relationships between spatially registered cortical parcellations under conditions of uncertainty. Neural Netw. 21, 1132-1145. doi:10.1016/j.neunet.2008.05.010

Blair, R. C., and Karniski, W. (1993). An alternative method for significance testing of waveform difference potentials. Psychophysiology 30, 518-524. doi:10.1111/j. 1469-8986.1993.tb02075.x

Boord, P., Siddall, P. J., Tran, Y., Herbert, D., Middleton, J., and Craig, A. (2008). Electroencephalographic slowing and reduced reactivity in neuropathic pain following spinal cord injury. Spinal Cord 46, 118-123. doi:10.1038/sj.sc.3102077

Brookes, M. J., Hale, J. R., Zumer, J. M., Stevenson, C. M., Francis, S. T., Barnes, G. R., et al. (2011). Measuring functional connectivity using MEG: methodology and comparison with fcMRI. Neuroimage 56, 1082-1104. doi:10.1016/j.neuroimage. 2011.02.054

Cheyne, D., Bakhtazad, L., and Gaetz, W. (2006). Spatiotemporal mapping of cortical activity accompanying voluntary movements using an event-related beamforming approach. Hum. Brain Mapp. 27, 213-229. doi:10.1002/hbm.20178

Cheyne, D., Bostan, A. C., Gaetz, W., and Pang, E. W. (2007). Event-related beamforming: a robust method for presurgical functional mapping using MEG. Neurophysiol. Clin. 118, 1691-1704. doi:10.1016/j.clinph.2007.05.064

Clarke, A. R., Barry, R. J., McCarthy, R., and Selikowitz, M. (2001). Age and sex effects in the EEG: development of the normal child. Neurophysiol. Clin. 112, 806-814. doi:10.1016/S1388-2457(01)00488-6 
Delorme, A., and Makeig, S. (2004). EEGLAB: an open source toolbox for analysis of single-trial EEG dynamics. J. Neurosci. Methods 134, 9-21. doi:10.1016/j. jneumeth.2003.10.009

Diaconescu, A. O., Alain, C., and McIntosh, A. R. (2011). The co-occurrence of multisensory facilitation and cross-modal conflict in the human brain. J. Neurophysiol. 106, 2896-2909. doi:10.1152/jn.00303.2011

Doesburg, S. M., Chau, C. M., Cheung, T. P., Moiseev, A., Ribary, U., Herdman, A. T., et al. (2013a). Neonatal pain-related stress, functional cortical activity and school-age cognitive outcome in children born at extremely low gestational age. Pain 152, 1946-1952. doi:10.1016/j.pain.2013.04.009

Doesburg, S. M., Vidal, J., and Taylor, M. J. (2013b). Reduced theta connectivity during set-shifting in children with autism. Front. Hum. Neurosci. 7:785. doi:10.3389/fnhum.2013.00785

Doesburg, S. M., Emberson, L. L., Rahi, A., Cameron, D., and Ward, L. M. (2008). Asynchrony from synchrony: long-range gamma-band neural synchrony accompanies perception of audiovisual speech asynchrony. Exp. Brain Res. 18, 11-20. doi:10.1007/s00221-007-1127-5

Doesburg, S. M., Ribary, U., Herdman, A. T., Moiseev, A., Cheung, T., Miller, S. P., et al. (2011a). Magnetoencephalography reveals slowing of resting peak oscillatory frequency in children born very preterm. Pediatr. Res. 70, 171-175. doi:10.1038/pr.2011.396

Doesburg, S. M., Ribary, U., Herdman, A. T., Miller, S. P., Poskitt, K. J., Moiseev, A., et al. (2011b). Altered long-range alpha-band synchronization during visual short-term memory retention in children born very preterm. Neuroimage 54, 2330-2339. doi:10.1016/j.neuroimage.2010.10.044

Dudink, J., Lequin, M., van Pul, C., Buijs, J., Conneman, N., van Goudoever, J., et al. (2007). Fractional anisotropy in white matter tracts of very-low-birth-weight infants. Pediatr. Radiol. 37, 1216-1223. doi:10.1007/s00247-007-0626-7

Frye, R. E., Malmberg, B., McLean, J., Swank, P., Smith, K., Papanicolaou, A., et al. (2010). Increased left prefrontal activation during an auditory language task in adolescents born preterm at high risk. Brain Res. 1336, 89-97. doi:10.1016/j. brainres.2010.03.093

Gonzalez, J. J., Manas, S., De Vera, L., Mendez, L. D., Lopez, S., Garrido, J. M., et al. (2011). Assessment of electroencephalographic functional connectivity in term and preterm neonates. Neurophysiol. Clin. 122, 696-702. doi:10.1016/j.clinph. 2010.08.025

Gross, J., Kujala, J., Hamalainen, M., Timmermann, L., Schnitzler, A., and Salmelin, R. (2001). Dynamic imaging of coherent sources: studying neural interactions in the human brain. Proc. Natl. Acad. Sci. U.S.A. 98, 694-699. doi:10.1073/pnas. 98.2.694

Grunau, R. E., Haley, D. W., Whitfield, M. F., Weinberg, J., Yu, W., and Thiessen, P. (2007). Altered basal cortisol levels at 3, 6, 8 and 18 months in infants born at extremely low gestational age. J. Pediatr. 150, 151-156. doi:10.1016/j.jpeds.2006. 10.053

Grunau, R. E., Whitfield, M. F., and Davis, C. (2002). Pattern of learning disabilities in children with extremely low birth weight and broadly average intelligence. Arch. Pediatr. Adolesc. Med. 156, 615-620. doi:10.1001/archpedi.156.6.615

Grunau, R. E., Whitfield, M. F., Petrie-Thomas, J., Synnes, A. R., Cepeda, I. L., Keidar, A., et al. (2009). Neonatal pain, parenting stress and interaction, in relation to cognitive and motor development at 8 and 18 months in preterm infants. Pain 143, 138-146. doi:10.1016/j.pain.2009.02.014

Hari, R., and Salmelin, R. (1997). Human cortical oscillations: a neuromagnetic view through the skull. Trends Neurosci. 20, 44-49. doi:10.1016/S0166-2236(96) 10065-5

Hart, A. R., Whitby, E. W., Griffiths, P. D., and Smith, M. F. (2008). Magnetic resonance imaging and developmental outcome following preterm birth: review of current evidence. Dev. Med. Child Neurol. 50, 655-663. doi:10.1111/j.1469-8749. 2008.03050.x

Hillebrand, A., Barnes, G. R., Bosboom, J. L., Berendse, H. W., and Stam, C. J. (2012). Frequency-dependent functional connectivity within resting-state networks: an atlas-based MEG beamformer solution. Neuroimage 59, 3909-3921. doi:10.1016/j.neuroimage.2011.11.005

Huang, M. X., Nichols, S., Robb, A., Angeles, A., Drake, A., Holland, M., et al. (2012). Integrated imaging approach with MEG and DTI to detect mild traumatic brain injury in military and civilian patients. Neuroimage 61, 1067-1082. doi:10.1089/neu.2008.0672

Huang, M. X., Theilmann, R. J., Robb, A., Angeles, A., Nichols, S., Drake, A., et al. (2009). Integrated imaging approach with MEG and DTI to detect mild traumatic brain injury in military and civilian patients. J. Neurotrauma 26, 1213-1226. doi:10.1089/neu.2008.0672

Hughes, S. W., and Crunelli, V. (2005). Thalamic mechanisms of EEG alpha rhythms and their pathological implications. Neuroscientist 11, 357-372. doi:10.1177/ 1073858405277450

John, E. R., Ahn, H., Prichep, L., Trepetin, M., Brown, D., and Kaye, H. (1980). Developmental equations for the electroencephalogram. Science 210, 1255-1258. doi:10.1126/science.7434026

Joliot, M., Ribary, U., and Llinás, R. (1994). Human oscillatory brain activity near $40 \mathrm{~Hz}$ coexists with cognitive temporal binding. Proc. Natl. Acad. Sci. U.S.A. 91, 11748-11751. doi:10.1073/pnas.91.24.11748

Khwaja, O., and Volpe, J. J. (2008). Pathogenesis of cerebral white matter injury of prematurity. Arch. Dis. Child. Fetal Neonatal Ed. 93, F153-F161. doi:10.1136/ adc. 2006.108837

Kostovic, I., and Judaš, M. (2010). The development of the subplate and thalamocortical connections in the human foetal brain. Acta Paediatr. 99, 1119-1127. doi:10.1111/j.1651-2227.2010.01811.x

Kötter, R., and Wanke, E. (2005). Mapping brains without coordinates. Philos. Trans. R. Soc. Lond. B Biol. Sci. 360, 751-766. doi:10.1098/rstb.2005.1625

Llinás, R., Urbano, F. J., Leznik, E., Ramirez, R. R., and van Marle, H. J. (2005). Rhythmic and dysrhythmic thalamocortical dynamics: GABA systems and the edge effect. Trends Neurosci. 28, 325-333. doi:10.1016/j.tins.2005.04.006

Llinás, R. R., Ribary, U., Jeanmonod, D., Kronberg, E., and Mitra, P. P. (1999). Thalamocortical dysrhythmia: a neurological and neuropsychiatric syndrome characterized by magnetoencephalography. Proc. Natl. Acad. Sci. U.S.A. 96, 15222-15227. doi:10.1073/pnas.96.26.15222

Marlow, N., Hennessy, E. M., Bracewell, M. A., Wolke, D., EPICure Study, and Group. (2007). Motor and executive function at 6 years of age after extremely preterm birth. Pediatrics 120, 793-804. doi:10.1542/peds.2007-0440

Mazaheri, A., Coffey-Corina, S., Mangun, G. R., Bekker, E. M., Berry, A. S., and Corbett, B. A. (2010). Functional disconnection of frontal cortex and visual cortex in attention-deficit/hyperactivity disorder. Biol. Psychiatry 67, 617-623. doi:10.1016/j.biopsych.2009.11.022

Ment, L. R., Hirtz, D., and Huppi, P. S. (2009). Imaging biomarkers of outcome in the developing preterm brain. Lancet Neurol. 8, 1042-1055. doi:10.1016/S14744422(09)70257-1

Miller, S. P., and Ferriero, D. M. (2009). From selective vulnerability to connectivity: insights from newborn brain imaging. Trends Neurosci. 32, 496-505. doi:10.1016/j.tins.2009.05.010

Miskovic, V., Schmidt, L. A., Boyle, M., and Saigal, S. (2009). Regional electroencephalogram (EEG) spectral power and hemispheric coherence in young adults born at extremely low birth weight. Neurophysiol. Clin. 120, 231-238. doi:10.1016/j.clinph.2008.11.004

Moiseev, A., Gaspar, J., Schneider, J., and Herdman, A. (2011). Application of multi-source minimum variance beamformers for reconstruction of correlated neural activity. Neuroimage 58, 481-496. doi:10.1016/j.neuroimage.2011. 05.081

Moiseev, A., and Herdman, A. (2013). Multi-core beamformers: derivation, limitations and improvements. Neuroimage 71, 135-146. doi:10.1016/j.neuroimage. 2012.12.072

Mulder, H., Pitchford, N. J., Hagger, M. S., and Marlow, N. (2009). Development of executive function and attention in preterm children: a systematic review. Dev. Neuropsychol. 34, 393-421. doi:10.1080/87565640902964524

Murias, M., Webb, S. J., Greenson, J., and Dawson, G. (2007). Resting state cortical connectivity reflected in EEG coherence in individuals with autism. Biol. Psychiatry 62, 270-273. doi:10.1016/j.biopsych.2006.11.012

Nevalainen, P., Pihko, E., Metsaranta, M., Andersson, S., Autti, T., and Lauronen, L. (2008). Does very premature birth affect the functioning of the somatosensory cortex? A magnetoencephalography study. Int. J. Psychophysiol. 68, 85-93. doi:10.1016/j.ijpsycho.2007.10.014

Okumura, A., Kubota, T., Tsuji, T., Kato, T., Hayakawa, F., and Watanabe, K. (2006). Amplitude spectral analysis of theta/alpha/beta waves in preterm infants. Pediatr. Neurol. 34, 30-34. doi:10.1016/j.pediatrneurol.2005.06.005

Palva, S., and Palva, J. M. (2007). New vistas for alpha-frequency band oscillations. Trends Neurosci. 30, 150-158. doi:10.1016/j.tins.2007.02.001

Palva, S., and Palva, J. M. (2012). Discovering oscillatory interaction networks with M/EEG: challenges and breakthroughs. Trends Cogn. Sci. (Regul. Ed.) 16, 219-230. doi:10.1016/j.tics.2012.02.004 
Papile, L. A., Burstein, J., Burstein, R., and Koffler, H. (1978). Incidence and evolution of subependymal and intraventricular hemorrhage: a study of infants with birth weights less than 1,500 gm. J. Pediatr. 92, 529-534. doi:10.1016/S0022-3476(78) 80282-0

Quraan, M. A., Moses, S. N., Hung, Y., Mills, T., and Taylor, M. J. (2011). Detection and localization of hippocampal brain activity using beamformers with MEG: a detailed investigation using simulations and empirical data. Hum. Brain Mapp. 32, 812-827. doi:10.1002/hbm.21068

Ribary, U. (2005). Dynamics of thalamo-cortical network oscillations and human perception. Progr. Brain Res. 150, 127-142. doi:10.1016/S0079-6123(05)50010-4

Rickards, A. L., Kelly, E. A., Doyle, L. W., and Callanan, C. (2001). Cognition, academic progress, behavior and self-concept at 14 years of very low birth weight children. J. Dev. Behav. Pediatr. 22, 11-18. doi:10.1097/00004703-20010200000002

Sarnthein, J., and Jeanmonod, D. (2007). High thalamocortical coherence in patients with Parkinson's disease. J. Neurosci. 27, 124-131. doi:10.1523/JNEUROSCI. 2411-06.2007

Sarnthein, J., and Jeanmonod, D. (2008). High thalamocortical coherence in patients with neurogenic pain. Neuroimage 41, 985-997. doi:10.1016/j.neuroimage.2007. 10.019

Sarnthein, J., Stern, J., Aufenberg, C., Rousson, V., and Jeanmonod, D. (2006). Increased EEG power and slowed dominant frequency in patients with neurogenic pain. Brain 129, 55-64. doi:10.1093/brain/awh631

Schnitzler, A., and Gross, J. (2005). Normal and pathological oscillatory communication in the brain. Nat. Rev. Neurosci. 6, 285-296. doi:10.1038/nrn 1650

Schnitzler, A., Münks, C., Butz, M., Timmermann, L., and Gross, J. (2009). Synchronized brain network associated with essential tremor as revealed by magnetoencephalography. Mov. Disord. 24, 285-296. doi:10.1002/mds.22633

Schulman, J. J., Cancro, R., Lowe, S., Lu, F., Walton, K. D., and Llinás, R. R. (2011). Imaging of thalamocortical dysrhythmia in neuropsychiatry. Front. Hum. Neurosci. 5:69. doi:10.3389/fnhum.2011.00069

Sekihara, K., Nagarajan, S., Poeppel, D., and Marantz, A. (2002). Performance of an MEG adaptive beamformer technique in the presence of correlated neural activities: effects on signal intensity and time-course estimates. IEEE Trans. Biomed. Eng. 49, 1534-1546. doi:10.1109/TBME.2002.805485

Sekihara, K., Nagarajan, S. S., Poeppel, D., Marantz, A., and Miyashita, Y. (2001). Reconstructing spatio-temporal activities of neural sources using an MEG vector beamformer technique. IEEE Trans. Biomed. Eng. 48, 760-771. doi:10.1109/ 10.930901

Smyser, C. D., Inder, T. E., Shimony, J. S., Hill, J. E., Degnan, A. J., Snyder, A. Z., et al. (2010). Longitudinal analysis of neural network development in preterm infants. Cereb. Cortex 20, 2852-2862. doi:10.1093/cercor/bhq035

Storey, J. D. (2002). A direct approach to false discovery rates. J. R. Stat. Soc. Series B Stat. Methodol. 64, 479-498. doi:10.1111/1467-9868.00346

Taylor, H. G., Minich, N. M., Klein, N., and Hack, M. (2004). Longitudinal outcomes of very low birth weight: neuropsychological findings. J. Int. Neuropsychol. Soc. 10, 149-163. doi:10.1017/S1355617704102038

Tokariev, A., Palmu, K., Lano, A., Metsaranta, M., and Vanhatalo, S. (2012). Phase synchrony in the early preterm EEG: development of methods for estimating synchrony in both oscillations and events. Neuroimage 60, 1562-1573. doi:10.1016/j.neuroimage.2011.12.080

Uhlhaas, P. J., Roux, F., Rodriguez, E., Rotarska-Jagiela, A., and Singer, W. (2010). Neural synchrony and the development of cortical networks. Trends Cogn. Sci. (Regul. Ed.) 14, 72-80. doi:10.1016/j.tics.2009.12.002

Uhlhaas, P. J., Roux, F., Singer, W., Haenschel, C., Sireteanu, R., and Rodriguez, E. (2009a). The development of neural synchrony reflects late maturation and restructuring of functional networks in humans. Proc. Natl. Acad. Sci. U.S.A. 106, 9866-9871. doi:10.1073/pnas.0900390106

Uhlhaas, P. J., Pipa, G., Lima, B., Melloni, L., Neuenschwander, S., Nikolic, D., et al. (2009b). Neural synchrony in cortical networks: history, concept and current status. Front. Integr. Neurosci. 3:17. doi:10.3389/neuro.07.017.2009

Valdés-Hernández, P. A., Ojeda-González, A., Martínez-Montes, E., LageCastellanos, A., Virués-Alba, T., Valdés-Urrutia, L., et al. (2010). White matter architecture rather than cortical surface area correlates with the EEG alpha rhythm. Neuroimage 49, 2328-2339. doi:10.1016/j.neuroimage.2009. 10.030

Varela, F., Lachaux, J. P., Rodriguez, E., and Martinerie, J. (2001). The brainweb: phase synchronization and large-scale integration. Nat. Rev. Neurosci. 2, 229-239. doi: $10.1038 / 35067550$

Ward, L. M. (2003). Synchronous neural oscillations and cognitive processes. Trends Cogn. Sci. (Regul. Ed.) 7, 553-559. doi:10.1016/j.tics.2003.10.012

Wechsler, D. (2003). Wechsler Intelligence Scales for Children (WISC-IV), 4th Edn. San Antonio: Psychological Corporation.

Wilson, H., Moiseev, A., Podin, S., and Quraan, M. (2007). Continuous head localization and data correction in MEG. Int. Congr. Ser. 1300, 623-626. doi:10.1016/j.ics.2007.02.051

Xiang, J., Liu, Y., Wang, Y., Kotecha, R., Kirtman, E. G., Chen, Y., et al. (2009). Neuromagnetic correlates of developmental changes in endogenous high-frequency brain oscillations in children: a wavelet-based beamformer study. Brain Res. 1274, 28-39. doi:10.1016/j.brainres.2009.03.068

Conflict of Interest Statement: The authors declare that the research was conducted in the absence of any commercial or financial relationships that could be construed as a potential conflict of interest.

Received: 12 June 2013; accepted: 30 October 2013; published online: 15 November 2013.

Citation: Doesburg SM, Moiseev A, Herdman AT, Ribary $U$ and Grunau RE (2013) Region-specific slowing of alpha oscillations is associated with visualperceptual abilities in children born very preterm. Front. Hum. Neurosci. 7:791. doi: 10.3389/fnhum.2013.00791

This article was submitted to the journal Frontiers in Human Neuroscience. Copyright (c) 2013 Doesburg, Moiseev, Herdman, Ribary and Grunau. This is an openaccess article distributed under the terms of the Creative Commons Attribution License (CC BY). The use, distribution or reproduction in other forums is permitted, provided the original author(s) or licensor are credited and that the original publication in this journal is cited, in accordance with accepted academic practice. No use, distribution or reproduction is permitted which does not comply with these terms. 